\author{
Simulated Probabilistic Population Estimation \\ Sherman Dorn \\ Arizona State University
}

\begin{abstract}
Author Note
Author email address: Sherman.dorn@asu.edu. This paper is available to the public on a CC-BY 4.0 International Creative Commons license; please cite generously.
\end{abstract}

Version 1.0. 2017 October 14. 


\begin{abstract}
One can estimate population sizes from random nonunique identifier variables such as birthdates or first names. Banks and Pandiani (2001) developed an efficient method of estimating population from the number of birthdates, and extended that to estimating overlaps of two populations. Banks and Pandiani took advantage of the (almost) uniform distribution of birthdates using the coupon-collector model in probability. This paper develops an alternative method from simulated data and extends the method to nonuniform distributions, such as names. The appendix provides applicable R code.
\end{abstract}




\section{Simulated Probabilistic Population Estimation}

To solve a practical problem in evaluating mental health policies and practices, Banks and Pandiani (2001, 2002, 2006) developed and patented a method of estimating single and overlapping populations from the number of birthdays present in a population, a method they called probabilistic population estimation (PPE). ${ }^{1}$ Because of the confidential nature of identity in health systems, Banks and Pandiani sought a tool that would allow the estimation of overlapping populations that would allow them and other researchers to answer critical questions for social-service agencies such as, How many recipients of services in a system also receive services in other systems? As they described, in evaluation it is more important to answer the how many question than the who question of record linkages.

Probabilistic population estimation works well when one has birthdate data and is working with populations small enough to avoid covering the entire range of birthdates in a year, or where other demographic data is available to stratify the populations into portions that do not cover all birthdates. In many cases, however, service agencies are reluctant to provide or work with birthdates, and for large data sets, even stratification leaves subsets covering the entire range of calendar dates in a year. ${ }^{2}$ But other potential identifiers (such as first name) have nonuniform distributions, and a simple extension of PPE is unworkable. Alternatives to PPE would help in those cases.

\section{Probabilistic Population Estimation}

Banks and Pandiani (2001) draw their approach from the probability area commonly known as the collector-coupon problem: if one is trying to collect a complete set of baseball

\footnotetext{
${ }^{1}$ The patents have since lapsed.

${ }^{2}$ The upper bound of the $95 \%$ confidence interval for the population with 364 birthdates is 3275 .
} 
cards or any other coupon or token, and one collects each coupon randomly from a uniform distribution, how many coupons must one draw to ensure a complete set? Banks and Pandiani realized that population size and birthdates is an analog to coupons drawn and types of coupons. If one assumes a uniform distribution of 365 birthdays in a year, ${ }^{3}$ and one only knows that a population has $d$ birthdates in it, the expected size of the population is

$$
N_{d}=\sum_{i=1}^{d} \frac{365}{365-i}[1]
$$

and the variance around that estimate is

$$
\sigma_{d}^{2}=\sum_{i=1}^{d} \frac{i * 365}{(365-i)^{2}}[2]
$$

For example, a population with 70 unique birthdates would have an expected population size of 77.8 individuals, and the $95 \%$ confidence interval ranges from 71.9 to 83.7 . This is a smaller potential error than alternative methods available at the time (Banks \& Pandiani, 2001).

\section{Simulating PPE}

One can simulate the coupon-collector problem by generating multiple iterations of a random draw from the underlying distribution of identifiers. One then uses the multiple estimates of the population size (one from each iteration) to estimate the expected population size as well as standard errors of that estimate. The steps in generating these estimates:

1. Identify an appropriate underlying distribution of identifiers. This might be uniform or near-uniform, as in birthdates within a single calendar year, or nonuniform, such as

\footnotetext{
${ }^{3}$ In their original work, Banks and Pandiani (2001) argue that the nonuniform distribution of birthdates in reality changes the estimates little. In simulations not shown in this paper, adding a $366^{\text {th }}$ birthdate with $1 / 4$ the distribution of all other dates also changes the probabilities little from the uniform-distribution formula.
} 
the most popular several hundred names given baby girls at birth in the United States in the 1990s (e.g., Galbi, n.d.). ${ }^{4}$

2. Set the number of unique identifiers $d$ that one is matching to a population size estimate.

3. Simulate a population by successively drawing from the underlying distribution with replacement.

4. For each draw of a single identifier, compare the number of unique identifiers in the simulated population (after the draw) to $d$.

5. Mark both the lowest and highest population sizes of the simulated population where the number of unique identifiers in the simulated population match $d$.

6. Repeat steps 3-5 many times - e.g., 1000 - and store the individual estimates from each iteration in a pool of estimates.

7. Fix the simulated estimate of the population size as the mean of the estimate pool created by step 6 , with appropriate standard error and other distributional characteristics from the pool's distribution of estimates.

After identifying an appropriate empirical distribution, the remainder is a straightforward process. The appendix provides code using the $\mathrm{R}$ language that calculates the estimates.

${ }^{4}$ According to the Social Security Administration, the most popular 1000 names generally cover between $70 \%$ and $75 \%$ of all birth names. The simulation here thus uses a truncated distribution. For practical purposes, a more complete applicable distribution would be advisable. 


\section{Simulated PPE vs. Formulaic PPE}

The simulated estimation of population is very close to the formulaic PPE described by Banks and Pandiani (2001). For example, for 70 unique identifiers, Banks and Pandiani's method estimates a population of 77.8 , with a standard deviation of the estimate as 3.0 . The simulated estimate is a population of 77.6 , with a standard deviation of 2.5 . The density plot of the pooled estimates appears as Figure 1, with the blue vertical line representing the pooled-estimate mean. More generally, estimates from simulations are both accurate and efficient; only 100 iterations is sufficient per $d$ unique birthdates to bring the simulated PPE to within $0.4 \%$ (on average) of the formulaic PPE, and the standard deviation of the pooled estimates within $2.3 \%$ (on average) of the formulaic standard error. Figure 2 demonstrates how closely the simulated population size estimates matches the formulaic estimates.

\section{Simulated PPE with Nonuniform Distributions}

The demonstrable advantage of simulated PPE is the capacity to estimate populations when the key identifier has a nonuniform distribution. The most popular 671 female names given baby girls in the United States during the 1990s form a test distribution for these purposes (Galbi, n.d.). The most popular name on the list, Christina, was the given name of 19,005 babies born in the 1990s and registered with the Social Security Administration, representing 2.88\% of babies given the most popular 671 names. Figure 3 shows the simulated PPE and 95\% confidentinterval bounds for $d=1$ to 100 unique identifiers ("names"). Figure 4 compares PPE estimates using both birthdates and 1990s girls' names. The nonuniform distribution of given names generates higher estimates of the population size than the birthdate (uniform) distribution. ${ }^{5}$

\footnotetext{
${ }^{5}$ If one used a 671-day calendar (i.e., compared, the population estimates are lower for each $d$. For example, with $d=70$, the formulaic PPE is 74.0.
} 


\section{Conclusion}

The uses of simulated PPE from nonunique persistent identifiers include both direct estimation of population sizes and indirect calculation of overlaps - i.e., sizes of populations were individuals matched on unique identifiers. As Banks and Pandiani (2001) explain, one can estimate not only separate populations but a merged group (or union of populations) from the merging of the identifiers such as birthdates or names, and thus infer the overlap. In a world where hacking of individual identities is common, the techniques that allow population estimation without knowing which individuals were in a population may seem quaint. It is still useful. 


\section{References}

Banks, S. M., \& Pandiani, J. A. (2001). Probabilistic population estimation of the size and overlap of data sets based on date of birth. Statistics in Medicine, 20(9-10), 1421-1430.

Banks, S. M., \& Pandiani, J. A. (2002). U.S. Patent No. 6,470,298. Washington, DC: U.S. Patent and Trademark Office.

Banks, S. M., \& Pandiani, J. A. (2006). U.S. Patent No. 7,139,675. Washington, DC: U.S. Patent and Trademark Office.

Galbi, D. (n.d.). Given name frequency project [website]. Retrieved from https://www.galbithink.org/names/agnames.htm. 


\section{Density estimate of population size with 70 birthdates}

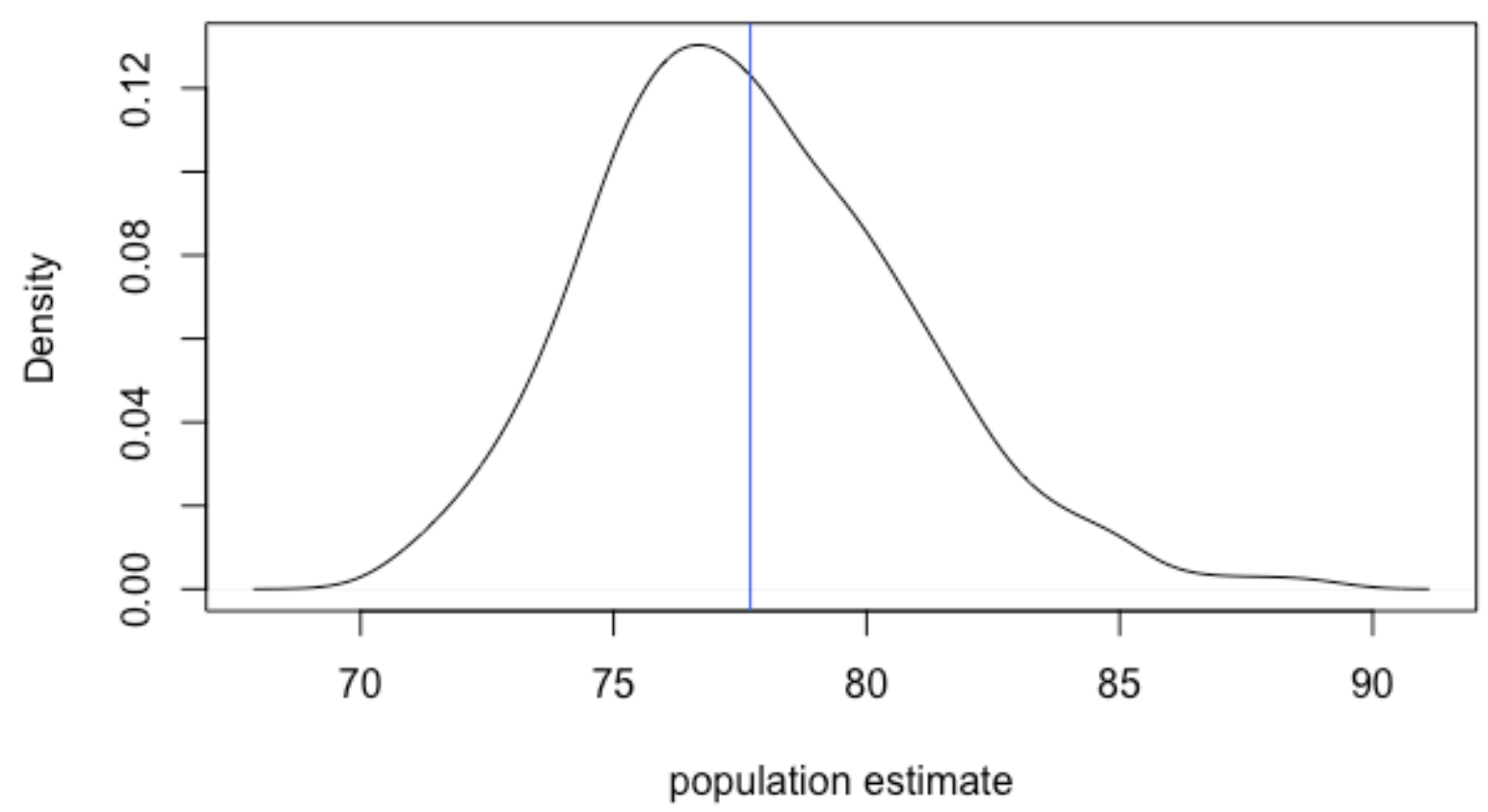

Figure 1. Density plot of pooled estimates of population size with 70 unique birthdates (uniform distribution of 365 birthdates in a year). Vertical line at 77.6 represents mean of simulated estimates. 


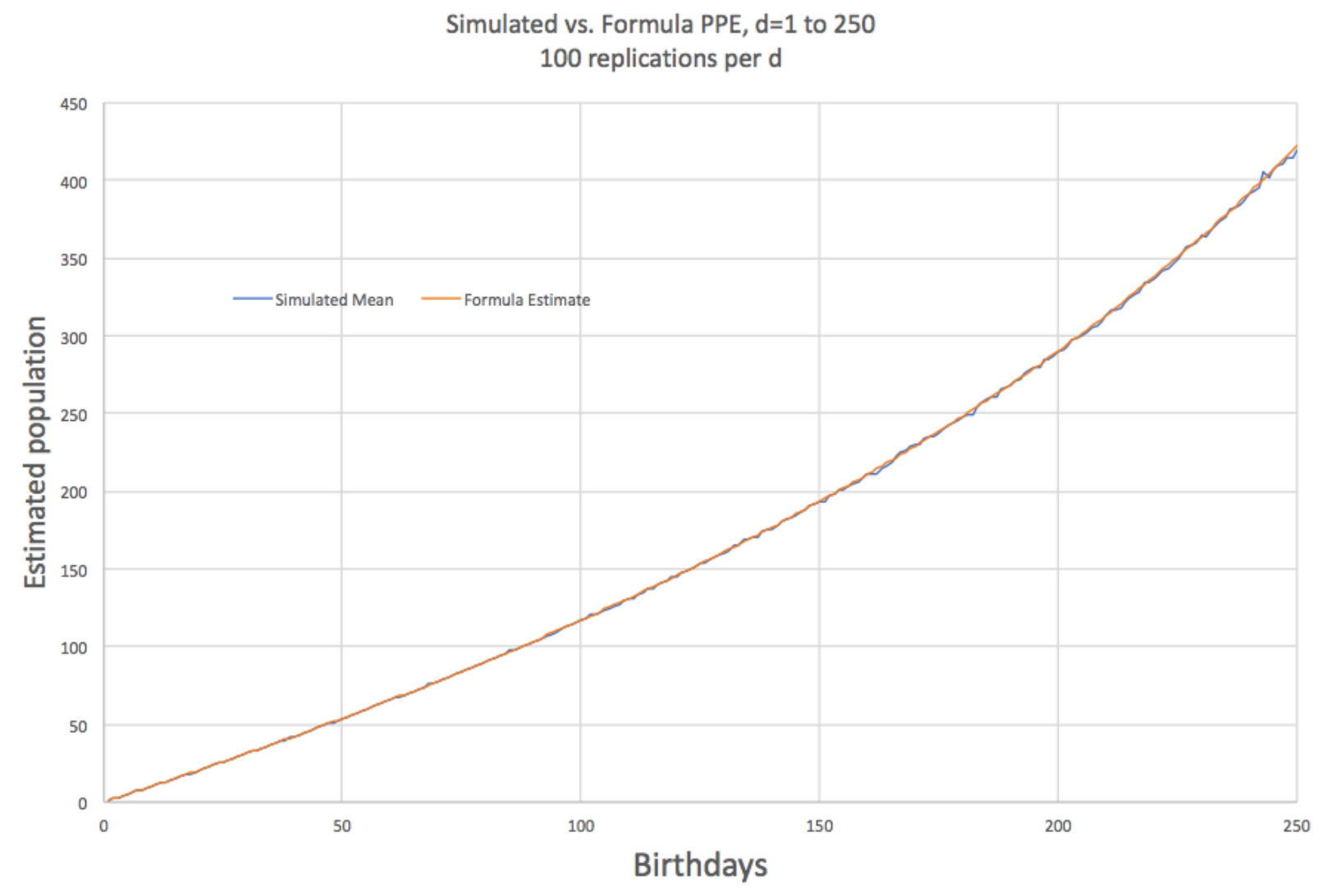

Figure 2. Comparing simulated PPE with formulaic PPE from Banks and Pandiani (2001), $d=1$ to 250 . This figure is generated from only 100 iterations per $d$. Average proportional bias is $0.4 \%$ of formulaic PPE. 


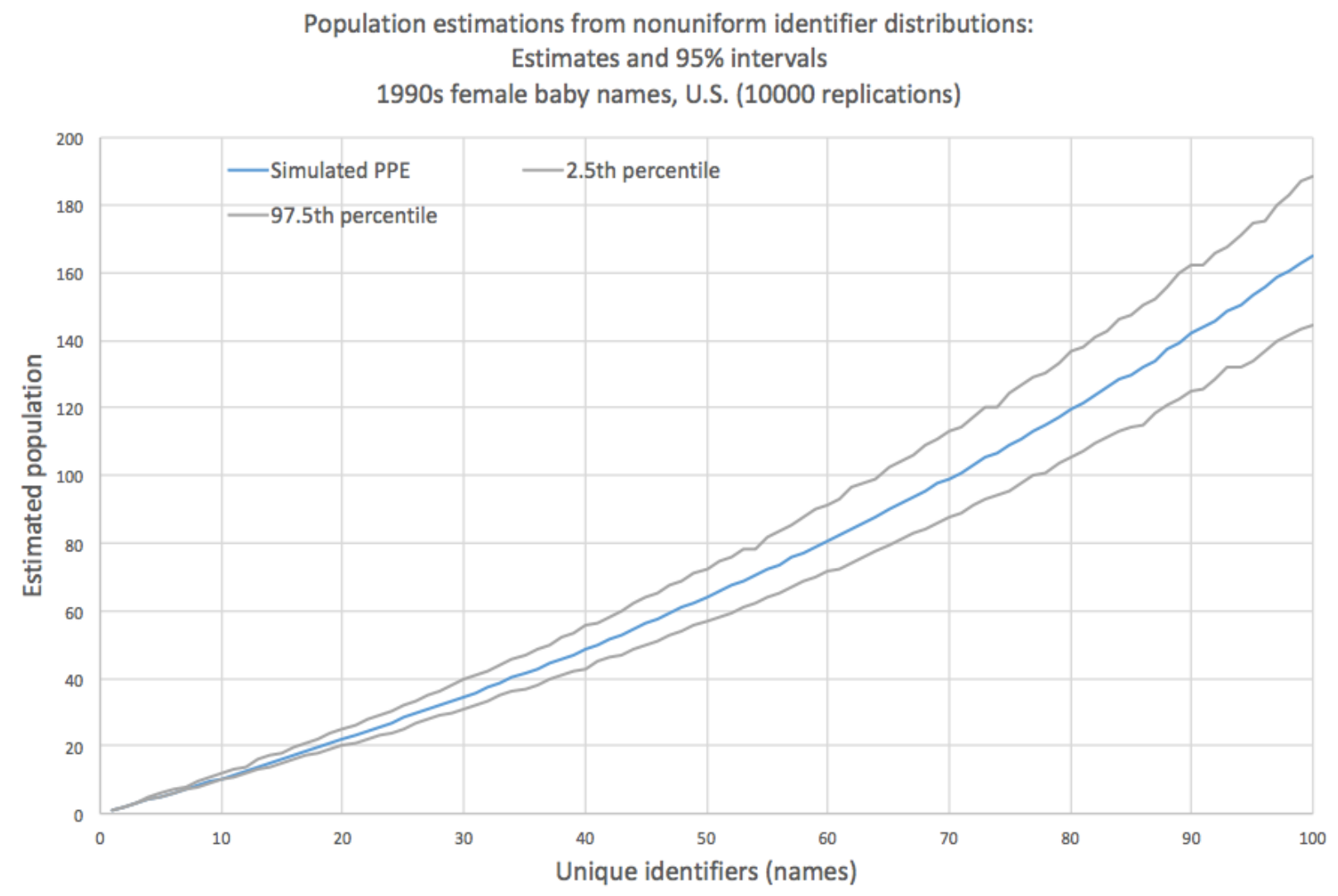

Figure 4. Simulated population estimation using the most popular girls' names in the United States in the 1990s, $d$ unique identifiers from 1 to 100. 
PPE by uniform vs nonuniform identifier distribution:

birthdates vs 1990 s female names in U.S.

(1000 replications per identifier for names)

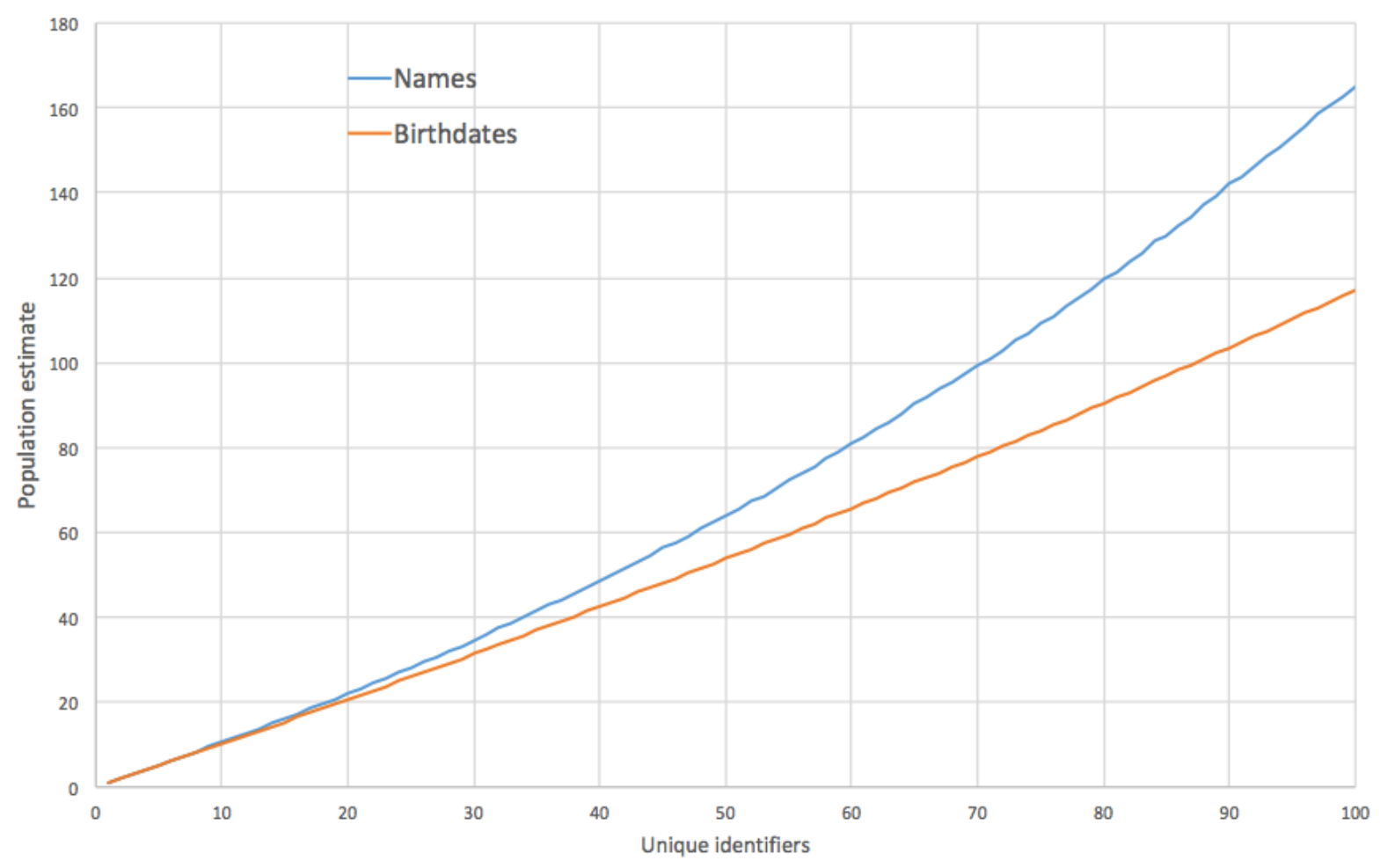

Figure 5. PPE estimates of population size for $d=1$ to 100 unique identifiers, using both birthdates (as uniform distribution) and 1990s girls' names (as nonuniform distribution) as examples. Simulated PPE for names, formulaic PPE for birthdates. 
Appendix

R Code for Simulated PPE

Below is code in the R language to estimate population sizes from empirical distributions of persistent, nonunique identifiers. The parameters of interest:

- uniques: $d$ in the formulae in the text, or the total number of unduplicated identifiers in a population of interest.

- edist: the table with the model distribution for the identifier in the population, with two columns, id (an arbitrary identifier label) and weight (probability in the relevant population).

- uniqueMax: the top number of unique identifiers where one wishes to calculate the estimated population.

- simulsPerUnique: how many simulated draws one wishes to calculate for each number of unique identifiers (e.g., 1000 or 10000).

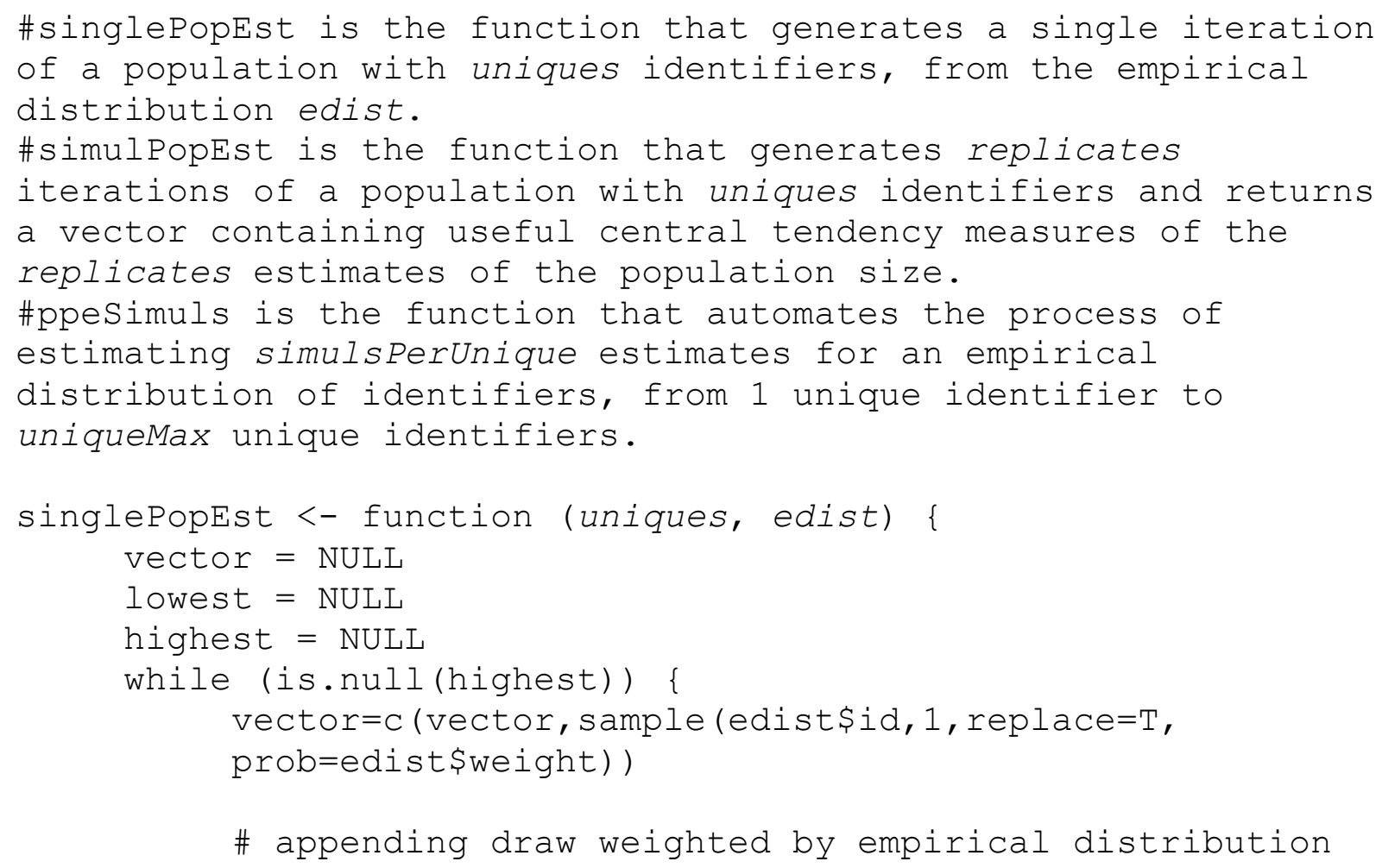




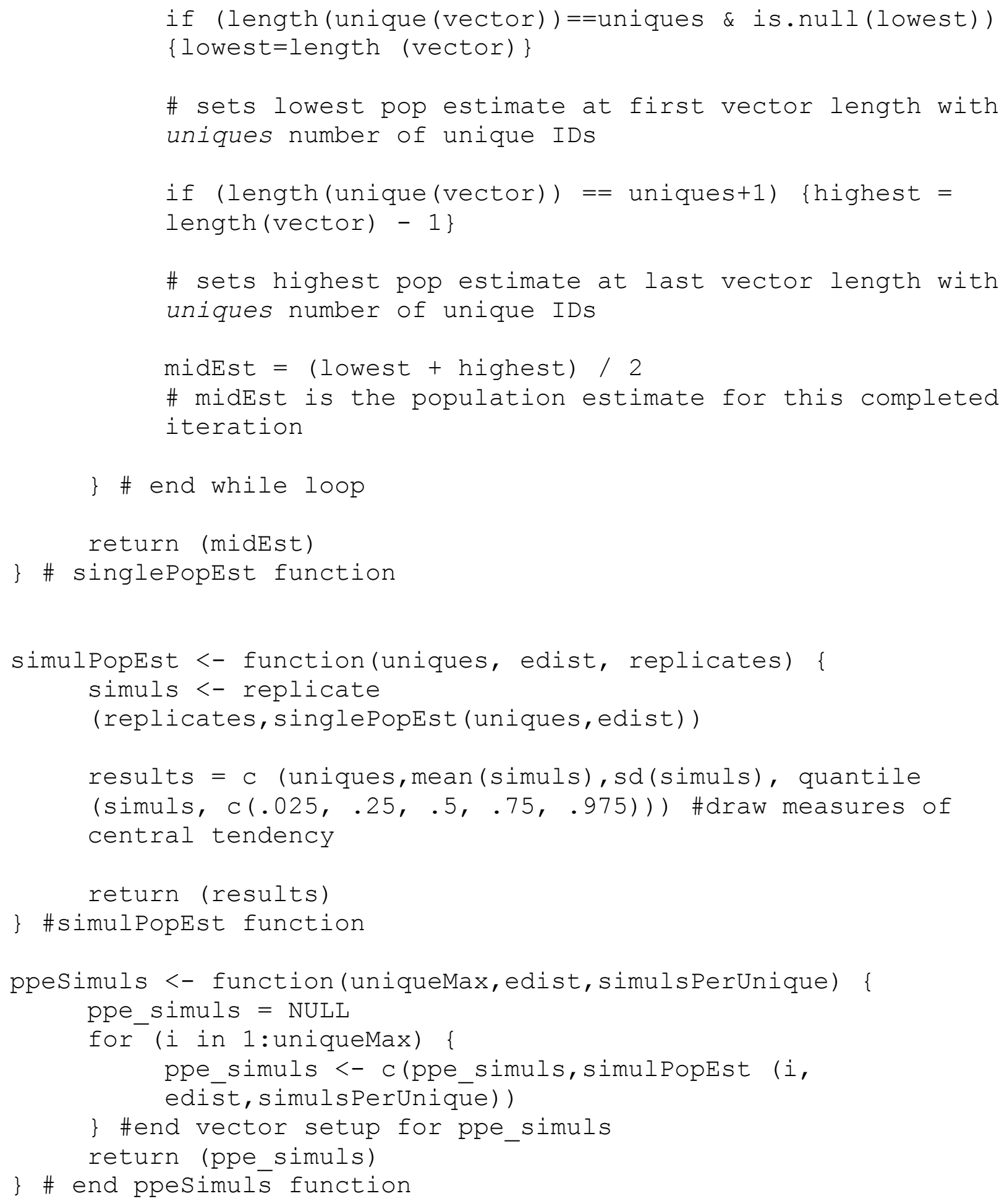

